\title{
Factors associated with unknown aetiology in patients with community-acquired pneumonia
}

\author{
S. Ewig*, A. Torres", M. Ángeles Marcos ${ }^{+}$, J. Angrill", A. Rañóo", A. de Roux", J. Mensa", \\ J.A. Martínez ${ }^{\uparrow}$, J.P. de la Bellacasa ${ }^{+}$, T. Bauer ${ }^{\S}$
}

Factors associated with unknown aetiology in patients with community-acquired pneumonia. S. Ewig, A. Torres, M.Ángeles Marcos, J. Angrill, A. Rañó, A. de Roux, J. Mensa, J.A. Martínez, J.P. de la Bellacasa, T. Bauer. (C) ERS Journals Ltd 2002. ABSTRACT: Despite comprehensive diagnostic work-up, the aetiology of communityacquired pneumonia (CAP) remains undetermined in $30-60 \%$ of cases. The authors studied factors associated with undiagnosed pneumonia.

Patients hospitalised with CAP and being evaluated by two blood cultures, at least one valid lower respiratory tract sample, and serology on admission were prospectively recorded. Patients who had received antimicrobial pretreatment were excluded. Patients with definite or probable aetiology were compared to those with undetermined aetiology by uni- and multivariable analysis.

A total 204 patients were eligible for the study. The aetiology remained undetermined in $82(40 \%)$ patients, whereas a definite aetiology could be established in $89(44 \%)$ and a probable one in $33(16 \%)$. In multivariable analysis, factors associated with undetermined aetiology included age $>70 \mathrm{yrs}$, renal and cardiac comorbidity, and nonalveolar infiltrates on the chest radiograph. There was no association of undiagnosed pneumonia with mortality.

Age and host factors were associated with unknown aetiology of community-acquired pneumonia. Some of these cases may also represent fluid volume overload mimicking pneumonia.

Eur Respir J 2002; 20: 1254-1262.

\begin{abstract}
*Medizinische Universitäts-Poliklinik Bonn, Germany. "\#Serveis de Pneumologia, "Malalties Infeccioses, ${ }^{+}$Microbiologia, Hospital Clinic Universitat de Barcelona, Spain. ${ }^{\S}$ Berugsgenossenschaftliche Klinik BergmannsheilUniversitätskliniken Innere Medizin, Bochum, Germany.
\end{abstract}

Correspondence: A. Torres, Hospital Clinic i Provincial, UVIR Servei de Pneumologia, Villarroel 170, 08036 Barcelona.

Fax: 34932275454

E-mail: atorres@medicina.ub.es

Keywords: Aetiology, epidemiology, pneumonia

Received: October 222001

Accepted after revision: June 52002

This study was supported by Commisionat per a Universitats i Recerca de la Generalitat de Catalunya,1997 SGR 00086, IDIBAPS Hospital Clinic Barcelona, and FISS 98/0138.
The aetiology of community-acquired pneumonia (CAP) has been studied in various regions and settings. While these studies differ considerably in patient populations, diagnostic methodology, and presence of confounders, one intriguing constant finding is the failure to detect a pathogen in $\sim 30-60 \%$ of cases [1-9]. Among the factors which may explain this observation, ambulatory antimicrobial pretreatment is the most attractive. Accordingly, there is evidence from the literature that the majority of cases of unknown aetiology may be caused by Streptococcus pneumoniae, a pathogen which is easily missed after one single dose of antimicrobial treatment [1,10]. Conversely, the recognition of Legionella pneumophila and Chlamydia pneumoniae has taught that unrecognised pathogens may represent important causes of CAP. Finally, comorbid conditions may represent important diagnostic confounders, either associated with distinct pathogens or as mimics of pneumonia.

A prospective study was therefore conducted in patients with CAP in order to identify factors associated with unknown aetiology. Since the authors were particularly interested in factors other than antimicrobial pretreatment, only previously untreated patients were selected. The prognostic implications of failure to detect microbial aetiology were also studied.

\section{Methods}

\section{Eligibility criteria}

All patients who presented to the emergency department of the Hospital Clinic I Provincial, a 1,000-bed tertiary care teaching hospital in Barcelona (Spain) during a 2-yr period were prospectively studied. Emergency care physicians were instructed to identify patients with pulmonary infiltrates and to report these patients immediately to a respiratory or infectious disease specialist on call for the purpose of this study.

In order to be eligible for this study, the following entry criteria had to be fulfilled: 1) CAP, as defined by a new infiltrate on the chest radiograph, symptoms compatible with a lower respiratory tract infection, and no alternative diagnosis emerging during follow-up; 2) absence of severe immunosuppression, as defined by a condition associated with a significant risk of opportunistic infection (solid organ or bone 
marrow transplantation, neutropenia $<1 \times 10^{9} \mathrm{~L}^{-1}$, treatment with oral corticosteroids in daily doses $\geqslant 20 \mathrm{mg}$ prednisolone-equivalent and/or $\geqslant 2$ weeks of treatment with azathioprine, cyclosporin or cyclophosphamide); 3) absence of human immunodeficiency virus (HIV) infection regardless of immune status; 4) the need for hospitalisation according to the judgment of the physician in charge; 5) the absence of previous hospitalisation for $>48 \mathrm{~h}$ within 30 days of the present hospital admission; 6) the absence of ambulatory antimicrobial pretreatment as defined by any antimicrobial treatment administered within 30 days of the present hospital admission; and 7) the presence of an aetiological work-up, consisting of two blood cultures, at least one valid lower respiratory tract sample, and serology on admission.

The following lower respiratory tract samples were accepted as valid: 1) sputum sample with $>25$ granulocytes and $<10$ epithelial cells per low power field total magnification $\times 100$ in the Gram stain; 2) protected specimen brush (PSB); 3) bronchoalveolar lavage (BAL); and 4) tracheobronchial aspirate (TBAS, retrieved via the endotracheal tube in intubated patients).

\section{Data recording}

The following parameters were recorded at admission: age, sex, smoking and alcohol habits, comorbidity, residence in nursing home, probable aspiration, current medication, clinical symptoms (cough, dyspnoea, chest pain, body temperature, respiratory rate, cardiac frequency, arterial systolic and diastolic blood pressure, blood gas analysis (oxygen and carbon dioxide tensions in arterial blood $\left(\mathrm{Pa}_{\mathrm{a}} \mathrm{O}_{2}, \mathrm{~Pa}_{\mathrm{a}} \mathrm{CO}_{2}\right)$, inspiratory oxygen fraction $\left.\left(F \mathrm{I}, \mathrm{O}_{2}\right)\right)$, chest radiograph pattern (alveolar, interstitial or mixed infiltrate, number of lobes affected, pleural effusion), and serum creatinine. At the clinical end-points of hospital discharge or death, the following parameters were additionally retrieved: definite microbial aetiology, type of sample the definite microbial diagnosis was based on (sputum, serology, antigen-detection, culture of blood or pleural effusion, and other lower respiratory tract specimen), antimicrobial treatment administered during hospital stay, admission to the intensive care unit (ICU), and 30 day in-hospital outcome.

\section{Definitions}

The following definitions were applicable to the study: 1) comorbid illnesses: cardiac (treatment for coronary artery disease or congestive heart failure or presence of valvular heart disease), pulmonary (treatment for asthma or chronic obstructive pulmonary disease or presence of interstitial lung disorders), renal (pre-existing renal disease with documented abnormal serum creatinine outside the pneumonia episode), hepatic (pre-existing viral or toxic liver disease), central nervous system disorders (presence of symptomatic acute or chronic vascular or nonvascular brain disease, with or without dementia), diabetes mellitus (intolerance to glucose and treatment with oral antidiabetics or insulin), neoplastic (any solid tumour active at the time of presentation or requiring antineoplastic treatment within the last year); 2) alcohol abuse: the ingestion of $>80 \mathrm{~g}$ of alcohol per day at least during the last year or prior abuse; 3 ) current smokers: those who smoked at least 10 cigarettes $\cdot$ day $^{-1}$ during the last year at least; 4) probable aspiration: witnessed aspiration or presence of risk factors for aspiration (severely altered consciousness, abnormal gag reflex or abnormal swallowing mechanism) [11]; 5) medication with $\mathrm{H}_{2}$-blockers: ranitidine or similar in a dose of at least $150 \mathrm{mg} \cdot$ day $^{-1}$ for at least 30 days prior to hospital admission; 6) severe sepsis or septic shock: systemic inflammatory response to infection (presence of $\geqslant 2$ of the following: temperature $>38^{\circ} \mathrm{C}$ or $<36^{\circ} \mathrm{C}$, cardiac frequency $>90$ beats $\cdot \mathrm{min}^{-1}$, respiratory rate $>20 \mathrm{~min}^{-1}$ or $P a, \mathrm{CO}_{2}<32 \mathrm{mmHg}$, and leucocyte count $>12,000 \mathrm{~mm}^{-3}$ or $>10 \%$ band forms) in addition to hypotension (systolic blood pressure $<90 \mathrm{mmHg}$ or diastolic blood pressure $<60 \mathrm{mmHg}$ ), with or without end organ damage [12]; and 7) acute respiratory failure: presence of respiratory rate $>30 \mathrm{breath} \cdot \mathrm{min}^{-1}, \mathrm{~Pa}, \mathrm{O}_{2} / F \mathrm{I}, \mathrm{O}_{2}<250$, or the requirement for mechanical ventilation.

\section{Microbiological evaluation}

Sampling included sputum, two blood cultures, and paired serology. Pleural puncture, TBAS, and flexible bronchoscopy with PSB or BAL were additional diagnostic techniques, applied according to the clinical judgment of the physician in charge. Urine samples were investigated for L. pneumophila serogroup I antigen titres.

Validated sputum, blood culture samples, pleural fluid, and undiluted and serially diluted TBAS, PSB, and BAL fluid samples were plated on the following media: blood-sheep agar, Centers for Disease Control agar, chocolate agar as well as Sabouraud agar. The identification of microorganisms was performed according to standard methods [13].

The aetiology was regarded to be probable when the causative organism was identified in sputum. It was considered to be definite if one of the following criteria were met: 1) blood cultures yielding a bacterial or fungal pathogen (in the absence of an apparent extrapulmonary focus); 2) bacterial growth in cultures of TBAS $\geqslant 10^{5}$ colony forming units (cfu) $\cdot \mathrm{mL}^{-1}$, in PSB $\geqslant 10^{3} \mathrm{cfu} \cdot \mathrm{mL}^{-1}$, and in BAL fluid $\geqslant 10^{4} \mathrm{cfu} \cdot \mathrm{mL}^{-1}$; 3) seroconversion (four-fold rise in immunoglobulin ( $\mathrm{Ig}) \mathrm{G}$-titres) for: $C$. pneumoniae $(\geqslant 1: 512)$, C. psittaci $(\geqslant 1: 64)$, L. pneumophila $(\geqslant 1: 128)$, Coxiella burnetii $(\geqslant 1: 80)$, respiratory viruses (Influenza virus $\mathrm{A}$ and $\mathrm{B}$, Parainfluenza virus $1-3$, respiratory syncytial virus, adenovirus); and 4) a rise in IgM-titre for: $C$. pneumoniae $(\geqslant 1: 32)$, C. burnetii $(\geqslant 1: 80)$, and Mycoplasma pneumoniae (any titre).

In addition to these criteria, pleural fluid cultures yielding a bacterial pathogen, and a positive urinary antigen for L. pneumophila were accepted as definitely diagnostic.

Growth of fungi in respiratory samples was only 
considered diagnostic in case of positive blood cultures for Candida spp., or isolation of Histoplasma capsulatum or Aspergillus fumigatus from lower respiratory tract cultures.

\section{Statistical analysis}

In a first step, all patients included in the analysis were grouped according to the aetiology into probable or definite aetiology or no aetiology according to the definitions given above. The second step involved univariable analysis for the candidate variables. An unpaired t-test was used for the comparison of quantitative variables between patient groups. Proportions were compared by the Chi-squared test or Fisher's exact test in comparisons with an expected cell frequency of less than five.

For multivariable analysis, a logistic regression model with forward stepwise selection was employed. The problems associated with multivariable analysis were addressed as follows. Stringent entry criteria $(\mathrm{p}<0.10$ in univariable analyses) reduced the number of candidate variables to avoid over-fitting. To minimise the possibility of including two highly correlated variables into the statistical model a stepwise forward model was chosen $(p<0.05)$. Interactions were analysed pairwise by entering an interaction term into the logistic regression analysis. Results are reported separately when interaction was found $(\mathrm{p}<0.05)$. All variables remained in their original dimension in the initial analysis to avoid bias induced by arbitrary cutoffs. Results of all multivariable analyses are reported as adjusted odds ratio, 95\% confidence intervals (CI) and exact p-values.
The initial multivariable model was repeated in a larger population that fulfilled all criteria except the completeness of the diagnostic sampling to control for bias induced by the selection criteria.

Results are expressed as mean \pm sD. All p-values presented refer to two-tailed analyses.

\section{Results}

\section{Patients}

Overall 719 episodes were entered into the initial database. The following patients were excluded: no or previous hospital admission $(n=50)$, severe immunosuppression $(n=47)$, HIV-infection $(n=50)$, alternative diagnosis $(\mathrm{n}=13)$, previous antimicrobial treatment $(n=135)$. The remaining population $(n=424)$ formed the larger population. Of these, the following had to be excluded additionally: no blood cultures at admission $(n=127)$, no valid respiratory tract sample $(n=72)$, no paired serology $(n=21)$. Thus, after the application of all exclusion criteria, 204 of 719 episodes were selected for this analysis $(28 \%)$.

\section{Aetiology}

Overall, a probable or definite aetiology could not be established in 82 of 204 included cases $(40 \%)$. The group of patients that remained without an aetiology was the largest single patient group.

The aetiologies as they were identified according to the given definitions as well as the corresponding diagnostic criteria are summarised in table 1.

Table 1.-Bacteriological findings of the 122 patients with either probable or definite aetiology of the episode of community-acquired pneumonia

\begin{tabular}{|c|c|c|c|c|c|c|c|}
\hline & Total & Sputum & $\begin{array}{l}\text { Blood } \\
\text { culture }\end{array}$ & TBAS & $\begin{array}{l}\text { PSB, } \\
\text { BAL }\end{array}$ & $\begin{array}{l}\text { Pleural } \\
\text { fluid }\end{array}$ & $\begin{array}{c}\text { Serology, } \\
\text { urinary antigen }\end{array}$ \\
\hline Streptococcus pneumoniae & 38/204 (19) & $19 / 38(50)$ & $17 / 38(45)$ & $1 / 38(50)$ & & $1 / 38(3)$ & \\
\hline Haemophilus influenzae & $12 / 204(6)$ & $9 / 12(75)$ & $2 / 12(17)$ & & & $1 / 12(8)$ & \\
\hline Moraxella catarrhalis & 2/204 (1) & $1 / 2(50)$ & & $1 / 2(50)$ & & & \\
\hline Staphylococcus aureus & 4/204 (2) & & $3 / 4(75)$ & $1 / 4(25)$ & & & \\
\hline Streptococcus viridans & $3 / 204(2)$ & & $3 / 3(100)$ & & & & \\
\hline Streptococcus pyogenes & $1 / 204(0.5)$ & & $1 / 1(100)$ & & & & \\
\hline Pseudomonas aeruginosa & 9/204 (4) & $3 / 9(33)$ & $1 / 9(11)$ & $3 / 3(33)$ & $2 / 9(22)$ & & \\
\hline Klebsiella pneumoniae & $1 / 204(0.5)$ & & $1 / 1(100)$ & & & & \\
\hline Escherichia coli & 4/204 (2) & & $3 / 4(75)$ & $1 / 4(25)$ & & & \\
\hline Chlamydia pneumoniae & $21 / 204(10)$ & & & & & & 21/21 (100) \\
\hline Chlamydia psittaci & $1 / 204(0.5)$ & & & & & & $1 / 1(100)$ \\
\hline Mycoplasma & $3 / 204(2)$ & & & & & & $3 / 3(100)$ \\
\hline Coxiella & $3 / 204$ (2) & & & & & & $3 / 3(100)$ \\
\hline Legionella pneumophila & $11 / 204(5)$ & & & & & & 11/11 (100) \\
\hline Mycobacterium tuberculosis & $1 / 204(0.5)$ & 1/1 (100) & & & & & \\
\hline Aspergillus fumigatus & $1 / 204(0.5)$ & & & $1 / 1(100)$ & & & \\
\hline Eikenella corrodens & $1 / 204(0.5)$ & & & & & $1 / 1(100)$ & \\
\hline Virus & 6/204 (3) & & & & & & $6 / 6(100)$ \\
\hline Total & $\begin{array}{c}204 / 204 \\
(100)\end{array}$ & $\begin{array}{c}33 / 122 \\
(27)\end{array}$ & $31 / 122(25)$ & $8 / 122(7)$ & $2 / 122(2)$ & $3 / 122(3)$ & $45 / 122(37)$ \\
\hline
\end{tabular}

Data are presented as $\mathrm{n} /$ total $\mathrm{n}(\%)$. Percentages in the first column refer to the total number of patients in the study ( $\mathrm{n}=204)$ to yield comparability to other diagnostics studies on community-acquired pneumonia. Probable: $n=33$; definite: $n=89$; community-acquired pneumonia: $\mathrm{n}=122$. TBAS: tracheobronchial aspirate (quantitative bacterial cultures $\geqslant 10^{5}$ colony forming units $\left.(\mathrm{cfu}) \cdot \mathrm{mL}^{-1}\right)$; PSB: protected specimen brush (quantitative bacterial cultures $\geqslant 10^{3} \mathrm{cfu} \cdot \mathrm{mL}^{-1}$ ); BAL: bronchoalveolar lavage. 
Table 2. - Demographic characteristics of patients with a successful diagnostic procedure compared to patients without aetiology

\begin{tabular}{|c|c|c|c|c|}
\hline Parameter & No aetiology & Probable or definite aetiology & p-value & $95 \% \mathrm{CI}$ of the difference \\
\hline Patients $n$ & $82 / 204(40)$ & $122 / 204(60)$ & & \\
\hline Age yrs mean $\pm s D$ & $71 \pm 18$ & $66 \pm 16$ & 0.019 & $1.0-0.4$ \\
\hline Sex male & $50 / 82(61)$ & $92 / 122(75)$ & 0.028 & $0.9-21.7$ \\
\hline Current smokers & $19 / 82(23)$ & $44 / 122(36)$ & 0.051 & $0.5-25.5$ \\
\hline Alcohol abuse & $8 / 82(10)$ & $25 / 122(21)$ & 0.041 & $1.3-20.7$ \\
\hline Nursing home admission & $4 / 81(5)$ & $4 / 121(3)$ & 0.560 & $-3.6-7.6$ \\
\hline Probable aspiration & $9 / 79$ (11) & $5 / 111(5)$ & 0.073 & $-2.0-14.0$ \\
\hline $\mathrm{H}_{2}$-blockers & $7 / 81(9)$ & $7 / 120(6)$ & 0.443 & $-4.5-10.5$ \\
\hline Comorbidity present & 65/82 (79) & $122 / 122(68)$ & 0.078 & $-1-1-23.1$ \\
\hline \multicolumn{5}{|l|}{ Type of comorbidity } \\
\hline Cardiac & 22/82 (27) & $12 / 122(10)$ & 0.001 & $6.0-28.0$ \\
\hline Pulmonary & $44 / 82(54)$ & $67 / 122(55)$ & 0.859 & $-12.9-4.9$ \\
\hline Chronic Bronchitis & $10 / 82(12)$ & $13 / 122(11)$ & 0.733 & $-8.0-0.0$ \\
\hline COPD & $29 / 82(35)$ & $38 / 122(31)$ & 0.529 & $-9.2-7.2$ \\
\hline Bronchiectasis & $0 / 82(0)$ & $3 / 122(3)$ & $0.211^{\#}$ & $0.0-6.0$ \\
\hline Asthma & $0 / 82(0)$ & $5 / 122(4)$ & $0.073^{\#}$ & $0.7-7.5$ \\
\hline Others & $5 / 82(6)$ & $8 / 122(7)$ & 0.918 & $-5.8-7.8$ \\
\hline Renal & $10 / 82(12)$ & $4 / 122(3)$ & 0.014 & $1.3-16.7$ \\
\hline Hepatic & $2 / 81(3)$ & $10 / 122(8)$ & 0.090 & $-1.1-1.1$ \\
\hline CNS & $12 / 82(15)$ & 15/122(12) & 0.888 & $-6.6-12.6$ \\
\hline Diabetes mellitus & $14 / 82(17)$ & $17 / 122(14)$ & 0.540 & $-7.2-13.2$ \\
\hline Neoplastic & $5 / 82(6)$ & $11 / 122(9)$ & 0.461 & $-4.2-10.2$ \\
\hline
\end{tabular}

Data are presented as $\mathrm{n} /$ total $\mathrm{n}(\%)$, unless otherwise stated. CI: confidence interval; COPD: chronic obstructive pulmonary disease; CNS central nervous system. ${ }^{\#}$ Fisher's exact test. The exact numbers are given for each parameter, since information was not available for all patients.

A probable aetiology was established in 33 of 122 cases $(27 \%)$. A definite aetiology was identified in 89 of 122 cases $(73 \%)$, based on serology or antigentesting in 45 of 122 cases $(37 \%)$, on blood cultures in 31 of 122 cases $(25 \%)$, on TBAS in eight of 122 cases $(7 \%)$, on pleural fluid in three of 122 cases $(3 \%)$ or on either PSB or BAL in two of 122 cases $(2 \%)$. No differences in the number of diagnostic procedures performed were found when patients with aetiology of their episode of CAP were compared to patients without: sputum (97 of $122(80 \%)$ versus 57 of 82 $(70 \%) ; \mathrm{p}=0.104)$, TBAS (19 of $122(16 \%)$ versus nine of $82(11 \%) ; \mathrm{p}=0.349)$, PSB, BAL (10 of $122(8 \%)$ versus four of $82(5 \%) ; p=0.358)$, and pleural fluid (12 of 122 $(10 \%)$ versus five of $82(6 \%) ; \mathrm{p}=0.343)$, respectively.

$S$. pneumoniae was identified as the causative microorganism in 38 of 204 patients (19\%), whereas "atypical" microorganisms (Chlamydia spp., Mycoplasma spp., or Coxiella spp.) were the second largest diagnostic group (28 of 204 patients, 14\%). H. influenzae was identified in 12 of 204 cases $(6 \%)$ and other Gram-negative bacteria (Pseudomonas aeruginosa, Klebsiella pneumoniae, or Escherichia coli) were recovered in 14 of 204 patients $(7 \%)$.

\section{Analysis of patients with unknown aetiology}

Demographic characteristics. The results of the comparison between patients with a probable or definite aetiology and patients without aetiology are summarised in table 2. Patients without aetiology were older (nondiagnostic: $71 \pm 18$ versus diagnostic: $66 \pm 16 \mathrm{yrs}, \mathrm{p}=0.019$ ), were less likely to be male (nondiagnostic: 50 of $82(61 \%)$ versus diagnostic:
92 of $122(75 \%), \mathrm{p}=0.028)$ and reported less alcohol abuse (nondiagnostic: eight of $82(10 \%)$ versus diagnostic: 25 of $122(21 \%), \mathrm{p}=0.041)$. There was a trend towards fewer smokers (nondiagnostic: 19 of 82 $(23 \%)$ versus diagnostic: 44 of $122(36 \%), \mathrm{p}=0.051)$ and more cases with probable aspiration (nondiagnostic: nine of $79(11 \%)$ versus diagnostic: five of $111(5 \%)$, $\mathrm{p}=0.073)$ in the group of patients with an undefined aetiology.

Comorbidity. The presence of a comorbidity seemed to lower the diagnostic yield (nondiagnostic: 65 of 82, $79 \%$ versus diagnostic: 83 of $122(68 \%), \mathrm{p}=0.078$; table 2). This was especially evident when the different types of comorbidities were analysed separately. A cardiac (nondiagnostic: 22 of $82(27 \%)$ versus diagnostic: 12 of $122(10 \%), \mathrm{p}=0.001)$ and renal comorbidity (nondiagnostic: 10 of $82(12 \%)$ versus diagnostic: four of $122(3 \%), p=0.014)$ were more frequent in the group of patients with unknown aetiology, whereas the diagnostic yield was higher in the group of patients with hepatic comorbidity (nondiagnostic: two of $81(3 \%)$ versus diagnostic: 10 of $122(8 \%), \mathrm{p}=0.090)$. A pulmonary comorbidity did not influence this comparison (nondiagnostic: 44 of $82(54 \%)$ versus diagnostic: 67 of $122(55 \%), \mathrm{p}=0.859)$.

Clinical data on admission and chest radiographs. No significant differences were present in the comparison of the clinical symptoms on admission. However, there was a nonsignificant trend towards a lower percentage of patients who reported pleuritic chest pain (nondiagnostic: 25 of 82 (31\%) versus diagnostic: 53 of $122(44 \%), p=0.056)$ or chills (nondiagnostic: 29 of 82 
Table 3.-Clinical characteristics and symptoms on admission of patients with a successful diagnostic procedure compared to patients without aetiology

\begin{tabular}{|c|c|c|c|c|}
\hline Parameter & No aetiology & $\begin{array}{c}\text { Probable or } \\
\text { definite aetiology }\end{array}$ & p-value & $\begin{array}{l}95 \% \text { CI of the } \\
\text { difference }\end{array}$ \\
\hline Patients $\mathrm{n}$ & $82 / 204(40)$ & $122 / 204(60)$ & & \\
\hline $\mathrm{Pa}, \mathrm{O}_{2} / F \mathrm{~F}, \mathrm{O}_{2} \mathrm{mmHg}$ & $262 \pm 66$ & $264 \pm 66$ & 0.840 & $-21.7-17.6$ \\
\hline Body temperature ${ }^{\circ} \mathrm{C}$ & $37.6 \pm 1.1$ & $37.8 \pm 1.1$ & 0.124 & $-0.5-0.1$ \\
\hline Respiratory rate bpm & $31.4 \pm 8.0$ & $30.0 \pm 9.6$ & 0.285 & $-1.2-4.0$ \\
\hline Duration of symptoms days & $4.5 \pm 4.0$ & $4.7 \pm 6.6$ & 0.751 & $-1.9-1.4$ \\
\hline Acute respiratory failure & $55 / 82(67)$ & $72 / 122(59)$ & 0.244 & $-5.4-21.4$ \\
\hline Septic shock & $12 / 82(15)$ & $28 / 122(23)$ & 0.142 & $-2.7-18.7$ \\
\hline Serum creatinine $\mathrm{mg} \cdot \mathrm{dL}^{-1}$ & $1.5 \pm 1.5$ & $1.2 \pm 0.8$ & 0.081 & $-0.1-0.6$ \\
\hline Serum albumin $\mathrm{mg} \cdot \mathrm{dL}^{-1}$ & $33.1 \pm 4.5$ & $33.1 \pm 7.4$ & 0.995 & $-2.0-2.0$ \\
\hline ICU admission & $9 / 82(11)$ & $20 / 122(16)$ & 0.277 & $-4.4-14.4$ \\
\hline Mechanical ventilation & $6 / 82(7)$ & $16 / 122(13)$ & 0.191 & $-2.1-14.1$ \\
\hline \multicolumn{5}{|l|}{ Type of radiological infiltrate } \\
\hline Bilateral & 28/82 (34) & $40 / 122(33)$ & 0.840 & $-12.2-14.2$ \\
\hline Alveolar & $48 / 78(62)$ & $91 / 121(75)$ & 0.040 & $-0.3-26.3$ \\
\hline Interstitial & $6 / 78(8)$ & $10 / 121(8)$ & 0.884 & $-7.7-7.7$ \\
\hline Mixed & $24 / 78(30)$ & 20/121 (17) & 0.018 & $0.8-25.2$ \\
\hline Pleural effusion & $14 / 79(18)$ & 16/116 (14) & 0.455 & $-6.6-14.6$ \\
\hline Cough & $63 / 82(78)$ & $92 / 122(75)$ & 0.697 & $-8.8-14.8$ \\
\hline Dyspnoea & 63/82 (77) & $84 / 122(69)$ & 0.213 & $-4.3-20.3$ \\
\hline Pleuritic chest pain & $25 / 82(31)$ & $53 / 122(44)$ & 0.056 & $-0.3-26.3$ \\
\hline Chills & $29 / 82(35)$ & $60 / 122(49)$ & 0.051 & $0.4-27.6$ \\
\hline $\begin{array}{l}\text { Preceding symptoms of } \\
\text { upper airways infections }\end{array}$ & $33 / 81(41)$ & $37 / 118(31)$ & 0.173 & $-3.6-23.6$ \\
\hline
\end{tabular}

Data are presented as $\mathrm{n} /$ total $\mathrm{n}(\%)$ or mean $\pm \mathrm{SD}$. CI: confidence interval; $P \mathrm{a}, \mathrm{O}_{2}$ : oxygen tension in arterial blood; $F \mathrm{I}, \mathrm{O}_{2}$ : inspiratory oxygen fraction; ICU: intensive care unit. Clinical characteristics and symptoms on admission of patients. The exact numbers are given for each parameter, since information was not available for all patients.

(35\%) versus diagnostic: 60 of $122(49 \%), \mathrm{p}=0.051)$ on admission in the nondiagnostic group (table 3 ). There was also a trend towards a higher serum creatinine in the undiagnosed group (nondiagnostic: $1.5 \pm 1.5$ versus diagnostic: $1.2 \pm 0.8 \mathrm{mg} \cdot \mathrm{dL}^{-1}, \mathrm{p}=0.081$; table 3 ).

The analysis of the radiographs on admission showed that alveolar appearance of the infiltrate was less common (nondiagnostic: 48 of $78(62 \%)$ versus diagnostic: 91 of $121(75 \%), \mathrm{p}=0.040)$, whereas a mixed pattern was significantly more often in the group of patients with undetermined aetiology (nondiagnostic: 24 of $78(30 \%)$ versus diagnostic: 20 of 121 $(17 \%), \mathrm{p}=0.018$; table 3$)$.

Multivariable analysis. In order to avoid interactions in the statistical model the authors did not include the presence of a comorbidity and serum creatinine because these confounding factors were covered by other variables (single comorbidities and renal comorbidity, respectively). According to the entry criteria for the multivariable analysis $(\mathrm{p}<0.10)$ the following 13 variables were then entered: age, sex, current smoker, alcohol abuse, probable aspiration, asthma, cardiac comorbidity, renal comorbidity, hepatic comorbidity, alveolar pulmonary infiltrate, mixed pulmonary infiltrate, pleuritic chest pain, and chills.

Overall, 21 cases had at least one missing value. Thus, 183 of $204(90 \%)$ of the patients could be included into the multivariable analysis (table 4). The risk of an episode of CAP without aetiology in the present study increased with age and the presence of a renal or cardiac comorbidity. The likelihood of unknown aetiology increased in the presence of something other than alveolar pulmonary infiltrate on the chest radiograph on admission (table 4).

Patients aged $\geqslant 70$ yrs had a two-fold risk of remaining without an aetiology when age was

Table 4.-Results of the multiple logistic regression analysis for qualified candidate variables

\begin{tabular}{|c|c|c|c|}
\hline & $\begin{array}{l}\text { Adjusted } \\
\text { odds ratio }\end{array}$ & p-value & $95 \% \mathrm{CI}$ \\
\hline Age (continuous) & $1.02^{\bullet}$ & 0.026 & $1.0-1.04$ \\
\hline $\begin{array}{l}\text { Renal } \\
\text { comorbidity }\end{array}$ & 5.1 & 0.057 & $1.0-26.7$ \\
\hline $\begin{array}{l}\text { Cardiac } \\
\text { comorbidity }\end{array}$ & 2.7 & 0.028 & $1.1-6.4$ \\
\hline $\begin{array}{l}\text { Infiltrate other } \\
\text { than alveolar }\end{array}$ & 2.5 & 0.008 & $1.3-5.0$ \\
\hline Age $\geqslant 70$ yrs & 2.0 & 0.033 & $1.1-3.9$ \\
\hline $\begin{array}{l}\text { Renal } \\
\text { comorbidity }\end{array}$ & 5.0 & 0.057 & $1.0-26.5$ \\
\hline $\begin{array}{l}\text { Cardiac } \\
\text { comorbidity }\end{array}$ & 2.9 & 0.014 & $1.2-6.9$ \\
\hline \multicolumn{4}{|l|}{ Interaction analysis ${ }^{\#}$} \\
\hline Age versus renal & & 0.114 & \\
\hline Age versus cardiac & & 0.004 & \\
\hline Age versus infiltrate & & 0.008 & \\
\hline Renal versus cardiac & & 0.110 & \\
\hline Renal versus infiltrate & & 0.657 & \\
\hline Cardiac versus infiltrate & & 0.055 & \\
\hline
\end{tabular}

CI: confidence interval. Adjusted odds ratios are reported for the risk of undiagnosed episode of community-acquired pneumonia. ${ }^{\#}$ : for categorised variable age; ": odds ratio for continuous variable. 
categorised for further analysis according to the group median (table 4). However, the effects of the other variables remained virtually unchanged by this measure. The interaction analysis revealed a significant interaction between age $\geqslant 70 \mathrm{yrs}$ and the presence of a cardiac comorbidity. This interaction is explained by the fact that the patients with both risk factors were more likely to remain without aetiology (nondiagnostic: 17 of $82(21 \%)$ versus diagnostic: eight of $122(7 \%), p=0.002)$. An age $\geqslant 70$ yrs also interacted with variables other than alveolar infiltrates. This interaction was also due to the disproportionally high percentage of patients with both risk factors in the undiagnosed group (nondiagnostic: 19 of $78(24 \%)$ versus diagnostic: 12 of $121(10 \%), \mathrm{p}=0.006)$.

Aetiology according to identified risk groups. Table 5 summarises the aetiological agents according to the risk groups identified in the multivariable analysis. The proportion of patients with an isolate of Moraxella catarrhalis or atypical agents was higher in the group of patients who presented with CAP in combination with a cardiac comorbidity. No other significant differences were found for this comparison.

Analysis in the larger population. A total of 424 of the 719 patients included in the database qualified for the larger population $(60 \%)$. In this group, 188 of 424 patients remained without probable or definite aetio$\operatorname{logy}(44 \%)$. Overall, 384 of $424(95 \%)$ were entered into the multivariable analysis. Cardiac comorbidity was the most important confounding factor for the sampling effort in this analysis (nondiagnostic: 52 of 188 $(28 \%)$ versus diagnostic: 31 of $236(13 \%), \mathrm{p}<0.001$, adjusted odds ratio: $2.6,95 \% \mathrm{CI}: 1.6-4.4, \mathrm{p}<0.001)$.

\section{Antimicrobial treatment}

Overall 175 patients (102 with and 73 without aetiology) were treated on the regular ward, and 29 (20 with and nine without aetiology) were admitted to the ICU. There were no significant differences in antimicrobial treatment regimens between patients with and without defined aetiology (table 6).
Outcome. The 30-day in-hospital mortality of patients with CAP included in this study was 12 of $204(6 \%)$, without differences between the diagnostic groups (nondiagnostic: three of $82(4 \%)$ versus diagnostic: nine of $122(7 \%), 95 \%$ CI $-3.2-9.2 ; \mathrm{p}=0.368$, Fisher's exact test). The 30-day in-hospital mortality in the larger population was undistinguishable from mortality in the investigated population (44 of $424(10 \%)$, $95 \%$ CI $-0.3-8.8 \% ; \mathrm{p}=0.824)$. No differences in mortality were found between the diagnostic groups in the larger population (nondiagnostic: 17 of $188(9 \%)$ versus diagnostic: 27 of $236(11 \%), 95 \% \mathrm{CI}:-3.7-7.7$; $\mathrm{p}=0.522)$.

\section{Discussion}

The main findings of this study were: 1) in the population investigated a total 82 of $204(40 \%)$ patients with CAP remained without aetiology, despite a substantial diagnostic effort; 2) multivariable analysis revealed age, renal and cardiac comorbidity, and nonalveolar radiological pattern as independent risk factors for an unknown aetiology; 3) the confounding role of cardiac comorbidity could be confirmed in a larger analysis; and 4) mortality was not different between patients with and without aetiology of CAP.

An important confounder which may have accounted for part of the undiagnosed cases is incomplete diagnostic work-up. A more extensive and aggressive diagnostic approach including antigen detection and polymerase chain reaction (PCR) may have increased the diagnostic yield. However, available evidence from the literature suggests that the diagnostic yield approaches a "ceiling effect" by $\sim 70-80 \%$ even when using a most comprehensive diagnostic approach [4, 6-8]. In the present study, it was ensured that every patient included was evaluated by at least two blood cultures, one valid sample of the lower respiratory tract, and serology, with additional testing performed according to clinical needs. This approach is unique in the literature since other studies included significant variations in the diagnostic testing applied to the individual patient. At the very least, the present study

Table 5. - Recovered microorganisms according to the risk factors identified in multivariate analysis

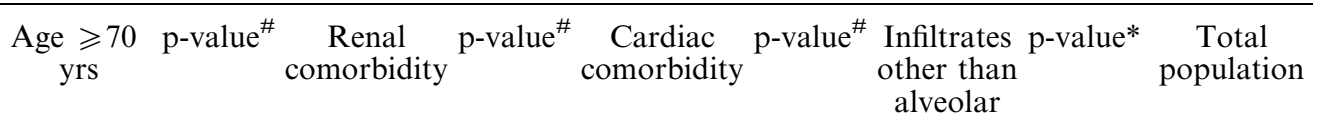

\begin{tabular}{|c|c|c|c|c|c|c|c|c|c|}
\hline With aetiology & $52 / 100(52)$ & & $4 / 14(29)$ & & $12 / 34(35)$ & & $30 / 60(50)$ & & $122 / 204(60)$ \\
\hline Streptococcus pneumoniae & $17 / 52(33)$ & 0.840 & $3 / 4(75)$ & $0.100^{\top}$ & $2 / 12(17)$ & $0.509^{\top}$ & $5 / 30(17)$ & 0.114 & $38 / 122(31)$ \\
\hline Haemophilus influenzae & $5 / 52(10)$ & 0.966 & $0 / 4(-)$ & 1.0 & $0 / 12(-)$ & $0.597^{\bullet}$ & $4 / 30(13)$ & 0.522 & $12 / 122(10)$ \\
\hline Moraxella catarrhalis & $1 / 52(2)$ & $1.0^{\circ}$ & $0 / 4(-)$ & $1.0^{\circ}$ & $2 / 12(17)$ & $0.046^{\oplus}$ & $0 / 30(-)$ & $1.0^{\circ}$ & $2 / 122(2)$ \\
\hline Gram-positive cocci $^{+}$ & $3 / 52(6)$ & $1.0^{\top}$ & $0 / 4(-)$ & $1.0^{\circ}$ & $1 / 12(8)$ & $0.581^{\bullet}$ & $2 / 30(7)$ & $1.0^{\circ}$ & $8 / 122(7)$ \\
\hline Gram-negative rods ${ }^{\S}$ & $5 / 52(10)$ & 0.718 & $0 / 4(-)$ & 1.0 & $0 / 12(-)$ & $0.367^{\bullet}$ & $3 / 30(10)$ & $1.0^{\top}$ & $14 / 122(12)$ \\
\hline Atypical $f^{\circ}$ & $10 / 51(20)$ & 0.628 & $1 / 4(25)$ & 1.0 & $6 / 12(50)$ & 0.049 & $8 / 30(27)$ & 0.668 & $28 / 122(23)$ \\
\hline Legionella pneumophila & $4 / 52(8)$ & $1.0^{\top}$ & $0 / 4(-)$ & 1.0 & $1 / 12(8)$ & $1.0^{\circ}$ & $4 / 30(13)$ & $0.479^{\circ}$ & $11 / 122(9)$ \\
\hline Others** & $4 / 52(8)$ & $1.0^{\bullet}$ & $0 / 4(-)$ & $1.0^{\top}$ & $0 / 12(-)$ & $1.0^{\bullet}$ & $4 / 30(13)$ & $0.287^{\bullet}$ & $9 / 122(7)$ \\
\hline
\end{tabular}

Data are presented as n/total n (\%). ${ }^{\#}$ : p-value compared to the larger population; ${ }^{\circ}$ : Fisher's exact test; ${ }^{+}:$Staphylococcus aureus, Streptococcus viridans, and Streptococcus pyogenes; ${ }^{\S}$ : Pseudomonas spp., Klebsiella pneumoniae, and Escherichia coli; f: Chlamydia spp., Coxiella spp., and Mycoplasma pneumoniae; **: Mycobacterium tuberculosis, Aspergillus fumigatus, Eikenella corrodens, and viruses. 
Table 6. - Initial antimicrobial treatment in patients with community-acquired pneumonia

\begin{tabular}{|c|c|c|c|}
\hline \multirow[t]{2}{*}{ Non-ICU patients } & \multicolumn{2}{|c|}{ Aetiology } & \multirow[t]{2}{*}{$\mathrm{p}$-value } \\
\hline & With & Without & \\
\hline Patients $\mathrm{n}$ & 102 & 73 & \\
\hline Monotherapy & $13(13)$ & $15(21)$ & 0.165 \\
\hline Aminopenicillin & 1 & 2 & 0.376 \\
\hline Aminopenicillin $\pm \beta$-lactamase inhibitor & & 5 & 0.007 \\
\hline Macrolide & 1 & 1 & 0.811 \\
\hline Cephalosporin III & 11 & 7 & 0.797 \\
\hline Dual combination therapy & $88(86)$ & $58(79)$ & 0.231 \\
\hline Cephalosporin III+macrolide & 80 & 50 & 0.138 \\
\hline Cephalosporin III+clindamycin & 5 & 6 & 0.372 \\
\hline Cephalosporin IV+macrolide & 1 & 1 & 0.811 \\
\hline Cephalosporin IV+aminoglycoside & 1 & 1 & 0.811 \\
\hline Carbapenem+aminoglycoside & 1 & & 0.396 \\
\hline Triple combination therapy & $1(1)$ & & 0.396 \\
\hline Cephalosporin III+macrolide+aminoglycoside & 1 & & 0.396 \\
\hline ICU patients $n$ & 20 & 9 & \\
\hline Dual combination therapy & $16(80)$ & $6(67)$ & 0.437 \\
\hline Cephalosporin III+macrolide & 14 & 6 & 0.857 \\
\hline Cephalosporin+clindamycin & 2 & & 0.325 \\
\hline Triple combination therapy & $4(20)$ & $3(33)$ & 0.437 \\
\hline Cephalosporin III+macrolide+aminoglycoside & 2 & 1 & 0.927 \\
\hline Cephalosporin III+macrolide+clindamycin & 1 & 1 & 0.547 \\
\hline Cephalosporin III+macrolide+vancomycin & 1 & & 0.494 \\
\hline Carbapenem + macrolide+aminoglycoside & & 1 & 0.129 \\
\hline
\end{tabular}

Data are presented as $\mathrm{n}(\%)$ or $\mathrm{n}$. Data are reported separately for patients with and without definite aetiology and according to intensive care unit admission (ICU). The p-values were calculated using a Chi-squared test.

succeeded in providing sufficiently homogeneous groups for comparison.

The rate of undiagnosed cases of CAP closely resemble those reported in prior studies e.g. two studies including $>500$ patients could not identify a pathogen in 50 and $45 \%$, respectively $[2,3]$. Likewise, in a previous study by the authors group, they failed to establish an aetiology in 54\% of pateints [9]. Without any doubt, prior antimicrobial treatment is an important factor which decreases the diagnostic yield. FANG et al. [14] clearly showed the decline in diagnostic yield in the presence of such treatment. In another large series, no diagnosis was made in $45 \%$ of the cases. With adjustment for antimicrobial therapy before admission and for other logistical considerations, it was estimated that the aetiology could have been ascertained in $65 \%$ of the cases [5]. The effect of antimicrobial treatment was analysed in more detail in the British Thoracic Society multicentre study. In this study, the rate of patients with evidence for pneumonia due to $S$. pneumoniae was more than twice as high in patients not given antimicrobial treatment before admission. Accordingly, $-55 \%$ of 150 cases with undetermined aetiology could have been accounted for by this pathogen [1]. Thus, it seems that most of the reduction in diagnostic yield by antimicrobial pretreatment was due to a failure to identify $S$. pneumoniae. This view is further supported by studies showing that using diagnostic techniques which are insensitive for antimicrobial pretreatment, such as antigen testing, increase the rate of pneumococcal aetiologies by $\leqslant 50 \%$. For example, in a British study, $63 \%$ of all pneumococcal aetiologies were exclusively established by pneumococcal antigen detection [15]. In a Dutch study, antigen was present in 12 of 25 patients with pneumonia of unknown aetiology who produced representative sputum [16]. Similar findings have also been reported by others [17, 18]. Therefore, the lack of antigen-testing represents a limitation of the present study. On the other hand, antigen detection is not an irrefutable diagnostic tool, and, therefore, does not mount definite evidence that positive testing in otherwise undiagnosed patients truly represents pneumococcal infection in every case. Corresponding considerations with regards to the role of $S$. pneumoniae in undiagnosed patients as well as limitations in accuracy are also true for new diagnostic tools such as PCR [19].

In the present study, the confounding factor of antimicrobial pretreatment was excluded. Nevertheless, it may still remain difficult to demonstrate a pneumococcal aetiology in nonbacteraemic pneumococcal pneumonia. Indirect evidence for a leading role of $S$. pneumoniae in undiagnosed patients was provided by a British study. In this report, the microbial aetiology was correctly predicted by a discriminant function analysis in $42 \%$ of cases. When a similar discriminant function analysis was applied to the one-third of patients in whom the microbial aetiology was never determined, most of these cases were predicted to be due to $S$. pneumoniae [10]. However, the present results suggest that after exclusion of the confounding factor of antimicrobial pretreatment, nonpneumococcal aetiologies and host factors play an important role in undiagnosed patients.

First, an unknown aetiology was more frequent in elderly patients, a cut-off of $70 \mathrm{yrs}$ being associated 
with a two-fold increase in risk. Studies addressing elderly patients with CAP tended to have undiagnosed cases at the higher extreme of the reported range (57 and $58 \%$, respectively) $[20,21]$. Accordingly, in a previous study by ÖRTQUIST et al. [4] age was also significantly associated with an undetermined aetiology. However, sputum, blood cultures, and paired sera were significantly less often obtained, and thus, the significance of this finding remained uncertain. The present results confirm the independent association of undetermined aetiology with age. This is an important finding, yet difficult to interpret. The most likely hypothesis is that elderly patients may be at higher risk for pathogens which are difficult to demonstrate. Among these, anaerobic pathogens involved in pneumonia due to silent (not gross) aspiration as well as viruses may be leading candidates. In fact, silent aspiration is a frequent event in elderly patients with CAP [22]. Moreover, these pathogens would be compatible with the nonalveolar radiographic pattern which was found to predominate in these patients.

Secondly, renal and cardiac comorbidity were independent predictors of an undetermined aetiology. Both volume overload due to renal failure as well as bronchial congestion due to congestive heart failure are risk factors for CAP [23]. However, whether these conditions predispose to specific pathogens is largely unknown. It was found that $M$. catarrhalis as well as atypical pathogens (C. pneumoniae, $M$. pneumoniae, and Coxiella spp.) were significantly associated with cardiac comorbidity. These pathogens may easily be missed by the usual diagnostic approach. Alternatively, part of this association may be due to the fact that volume overload may mimic pneumonia. This may occur not only by confounding radiographic findings but also by a noninfectious inflammatory response to bronchial congestion. However, only approximately one-third of the infiltrates were bilateral in both groups, which substantially reduces the probability that volume overload was the leading cause of failure to obtain an aetiology. Conversely, parameters other than those specified such as echocardiography- or nonpneumonia-related treatment were not recorded, these may have provided additional evidence for the relative role of fluid overload. This issue clearly should be assessed more specifically in a future study.

Finally, the nonalveolar radiographic pattern predominating in patients with undetermined aetiology strongly hints at pathogens other than pyogenic bacteria. LEVy et al. [24] demonstrated that alveolar patterns were due to pyogenic bacteria in $>90 \%$ of cases, whereas mixed patterns had a very heterogeneous aetiology including atypical bacterial and viral pathogens.

The role of diagnostic work-up in terms of outcome of CAP remains controversial. So far, no study has demonstrated a beneficial effect of establishing an aetiology on any outcome measure [25]. The only hint, with regards to the advantage of diagnostic tools, originates from a study of elderly patients. In this study, the outcome was better when blood cultures were performed within $24 \mathrm{~h}$ of admission [26].
However, for this finding to be conclusive there should be an excess mortality in patients with undetermined aetiology. In the present study, although antimicrobial treatment was comparable in patients with and without defined aetiology, a corresponding difference was not found, and the authors were not aware of any other study which might have found such an association.

The intention of this study was hypothesis generating and the results presented have to be interpreted accordingly. The statistical methods employed yielded results that were relevant to the population investigated therefore, they may change in different samples [27]. In order to give the reader an idea about the stability of the conclusions reached on a selected sample, the analyses were repeated in a large less selected population (referred to as the larger population) with the same results. This fact together with the clinical plausibility of the results presented supports the importance of the hypothesis and should stimulate future studies on the repeatibility of the predictors presented.

To conclude, age and host factors were found to be independent predictors of undetermined aetiology in community-acquired pneumonia. The predominance of a nonalveolar pattern in chest radiographs, leads the authors to believe that most undiagnosed cases may be due to pathogens which may be difficult to determine, particularly anaerobes involved in silent aspiration and atypical pathogens. Some of these cases may also have represented volume overload mimicking pneumonia. Although excess mortality in undiagnosed patients was not found, further efforts should be made to test the hypothesis generated from this study.

\section{References}

1. British Thoracic Society. Community-acquired pneumonia in adults in British hospitals in 1982-1983: a survey of aetiology, mortality, prognostic factors, and outcome. Q J Med 1987; 239: 195-220.

2. Marrie TJ, Dunant H, Yates L. Community-acquired pneumonia requiring hospitalization: 5-year prospective study. Rev Infect Dis 1989; 11: 586-599.

3. Blanquer J, Blanquer R, Borras R, et al. Aetiology of community acquired pneumonia in Valencia, Spain: a multicentre propspective study. Thorax 1991; 46: 508 511.

4. Örtquist A, Hedlund J, Grillner L, et al. Aetiology, outcome and prognostic factors in patients with community-acquired pneumonia requiring hospitalization. Eur Respir J 1990; 3: 1105-1113.

5. Bohte R, van Furth R, van den Broek PJ. Aetiology of community-acquired pneumonia: a prospective study among adults requiring admission to hospital. Thorax 1995; 50: 543-547.

6. Mundy LM, Auwaerter PG, Oldach D, et al. Community-acquired pneumonia: impact of immune status. Am J Respir Crit Care Med 1995; 152: 13091315.

7. Lieberman D, Schlaeffer F, Boldur I, et al. Multiple pathogens in adult patients admitted with communityacquired pneumonia: a one year propspective study of 346 consecutive patients. Thorax 1996; 51: 179-184. 
8. Neill AM, Martin IR, Weir R, et al. Community acquired pneumonia: aetiology and usefulness of severity criteria on admission. Thorax 1996; 51: 1010-1016.

9. Ruiz M, Ewig S, Marcos MA, et al. Aetiology of community-acquired pneumonia: impact of age, comorbidity, and severity. Am J Respir Crit Care Med 1999; 160: 397-405.

10. Farr BM, Kaiser DL, Harrison BDW, Connolly C. Prediction of microbial aetiology at admission to hospital for pneumonia from the presenting clinical features. Thorax 1989; 44: 1031-1035.

11. Leroy $\mathrm{O}$, Vandenbussche $\mathrm{C}$, Coffinier $\mathrm{C}$, et al. Community-acquired aspiration pneumonia in intensive care units. Epidemiological and prognosis data. Am J Respir Crit Care Med 1997; 156: 1922-1929.

12. Bone RC, Balk RA, Cerra FB, et al. Definitions for sepsis and organ failure and guidelines for the use of innovative therapies in sepsis. Chest 1992; 101: 1644 1655.

13. Balows A, Harsler WJ Jr. Manual of Clinical Microbiology. 5th edn. Washington DC, American Society for Microbiology, 1991; pp. 209-553.

14. Fang GD, Fine M, Orloff J, et al. New and emerging etiologies for community-acquired pneumonia with implications for therapy. A prospective multicenter study of 359 cases. Medicine 1990; 69: 307-316.

15. Macfarlane JT, Finch RG, Ward MJ, Macrae AD. Hospital study of adult community-acquired pneumonia. Lancet 1982; II: 255-258.

16. Boersma WG, Lowenberg A, Holloway Y, Kuttschrutter H, Snijder JA, Koeter GH. Pneumococcal capsular antigen detection and pneumococcal serology in patients with community acquired pneumonia. Thorax 1991; 46: 902-906.

17. Ruiz-Gonzalez A, Falguera M, Nogues A, RubioCaballero M. Is Streptococcus pneumoniae the leading cause of pneumonia of unknown aetiology? A microbiologic study of lung aspirates in consecutive patients with community-acquired pneumonia. $\mathrm{Am}$ J Med 1999; 106: 385-390.
18. Bella F, Tort J, Morera MA, Espaulella J, Armengol J. Value of bacterial antigen detection in the diagnostic yield of transthoracic needle aspiration in severe community-acquired pneumonia. Thorax 1993; 48: 1227-1229.

19. Menendez R, Cordoba J, de La Cuadra P, et al. Value of the polymerase chain reaction assay in noninvasive respiratory samples for diagnosis of communityacquired pneumonia. Am J Respir Crit Care Med 1999; 159: 1868-1873.

20. Venkatesan P, Gladman J, Macfarlane JT, et al. A hospital study of community acquired pneumonia in the elderly. Thorax 1990; 45: 254-258.

21. Riquelme $\mathrm{R}$, Torres $\mathrm{A}$, El-Ebiary $\mathrm{M}$, et al. Community-acquired pneumonia in the elderly. A multivariate analysis of risk and prognostic factors. Am J Respir Crit Care Med 1996; 154: 1450-1455.

22. Kikuchi R, Watabe N, Konno T, Mishina N, Sekizawa K, Sasaki H. High incidence of silent aspiration in elderly patients with communityacquired pneumonia. Am J Respir Crit Care Med 1994; 150: 251-253.

23. Farr BM, Woodhead MA, Macfarlane JT, et al. Risk factors for community-acquired pneumonia diagnosed by general practitioners in the community. Respir Med 2000; 94: 422-427.

24. Levy M, Dromer F, Brion N, Leturdu F, Carbon C. Community-acquired pneumonia. Importance of initial noninvasive bacteriologic and radiographic investigations. Chest 1988; 92: 43-48.

25. Sanyal S, Smith PR, Saha AC, Gupta S, Berkowitz L, Homel P. Initial microbiologic studies did not affect outcome in adults hospitalized with communityacquired pneumonia. Am J Respir Crit Care Med 1999; 160: 346-348.

26. Meehan TP, Fine MJ, Krumholz HM, et al. Quality of care, process, and outcomes in elderly patients with pneumonia. JAMA 1997; 278: 2080-2084.

27. Concato J, Feinstein AR, Holford TR. The risk of determining risk with multivariate models. Ann Intern Med 1993; 118: 201-210. 